\title{
Nonlinear Laser Lithography for Enhanced Tribological Properties
}

\author{
Iaroslav Gnilitskyi ${ }^{1}$, Ihor Pavlov ${ }^{2}$, Fabio Rotundo ${ }^{3}$, Leonardo Orazi ${ }^{1}$, Carla Martini ${ }^{3}$ and Fatih Ömer Ilday ${ }^{2,4}$ \\ 1 - Department of Sciences and Methods for Engineering - University of Modena and Reggio Emilia - Italy \\ 2 - Department of Physics - Bilkent University - Turkey \\ 3 - Industrial Engineering Department - University of Bologna - Italy \\ 4 - Department of Electrical and Electronics Engineering - Bilkent University - Turkey
}

iaroslav.gnilitskyi@unimore.it

\begin{abstract}
This paper investigates a new field for application of femtosecond laser-induced periodic surface structures (LIPSS). We designed an innovative solution to reduce coefficient of friction of mechanical parts by using the nonlinear laser lithography technique (NLL).

OCIS codes: (350.0350) General; (350.3850)
\end{abstract}

\section{Introduction}

In recent years, the production of nanostructures on different materials has attracted many attention by the reseachersdue to the availability of laser-based methods in different fields of manufacturing $[1,2]$. However, some limitations as low-speed, problems of material control, and lack of uniformity and repeatability over large areas prevented to use them for practical applications.

Recently, a technique called Nonlinear Laser Lithography (NLL) was introduced, which allows fabrication of extremely uniform nanostructures, with excellent long-range repeatability and high speed and productivity [3]. NLL could be applied to a variety of materials, including non-planar, even flexible surfaces. NLL generates essentially LIPSS-type nanostructures by utilizing nonlinear feedback mechanisms arising from the interaction of femtosecond laser pulses with the target surface, as well as from the laser-initiated chemical reactions. Key features, such as superior uniformity and ability to process non-flat surfaces are a direct consequence of the self-regulation provided by these feedback mechanisms. In the present work, a custom-built Yb-doped fiber laser system was used to provide a low cost method for the fabrication of large area microtextured surfaces. In particular, highly uniform NLL have been obtained on the AISI 316L stainless steel thanks to the choice of the optimal laser and scanning parameters.

AISI 316L Stainless Steel was treated in ambient atmosphere using a femtosecond fiber laser. NLL was used to obtain homogeneous LIPSS on large surface areas (up to $50 \mathrm{~mm}^{2}$ ). The tribological properties of the treated surfaces were evaluated by both unlubricated and lubricated sliding tests, considering different orientations of the lasertextured surface with respect to the sliding direction. The results showed a significant decrease of the friction coefficient for LIPSS treated surfaces, thanks to the induced nanostructures. The results shown that NLL is effective in reducing the maximum Coefficient of Friction ( $\mathrm{COF}$ ) both in lubricated and unlubricated conditions in the range from 50 to $80 \%$.

In the present work, the tribological behavior of laser textured AISI 316L was assessed by sliding tests, carried out both in lubricated (ball-on-disk contact geometry) and dry (block-on-ring) conditions. The results allowed to evaluate the tribological behaviour of femtosecond laser-induced periodic surface structures on AISI 316 L austenitic stainless steel as a function of the following variables: (i) orientation of the LIPSS pattern with respect to the sliding direction (parallel/perpendicular lines/perpendicular lines with stripes), (ii) nature of the countermaterial (in dry sliding conditions), (iii) absence or presence of lubrication. The results shown that NLL is effective in reducing the maximum Coefficient of Friction ( COF ) both in lubricated and unlubricated conditions in the range from 50 to $80 \%$. 


\section{Figures}

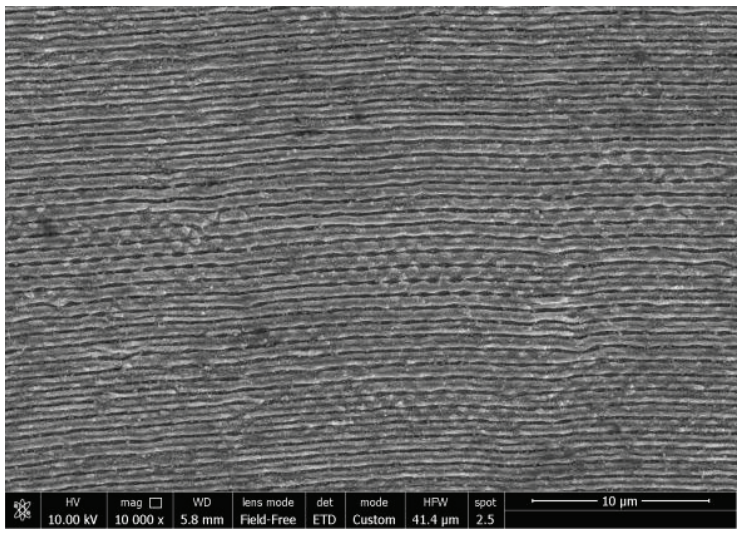

$\mathrm{a}$

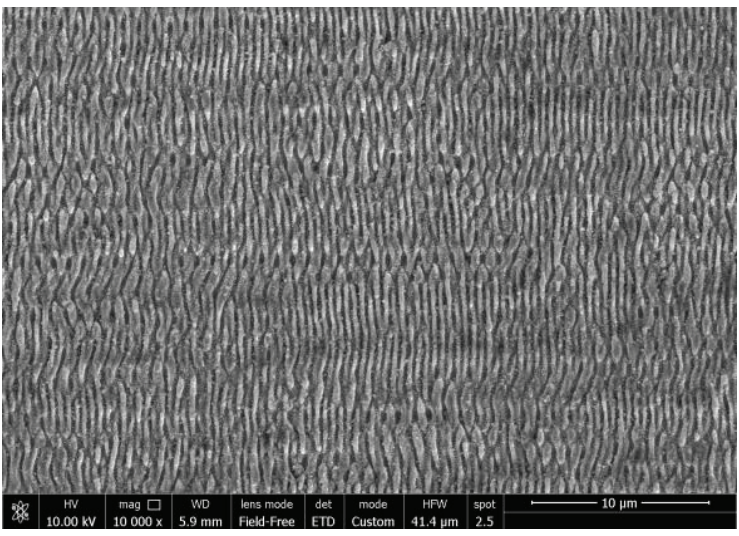

b

Fig. 1. NLL structures with perpendicular (a) and parallel (b) direction to counterbody.

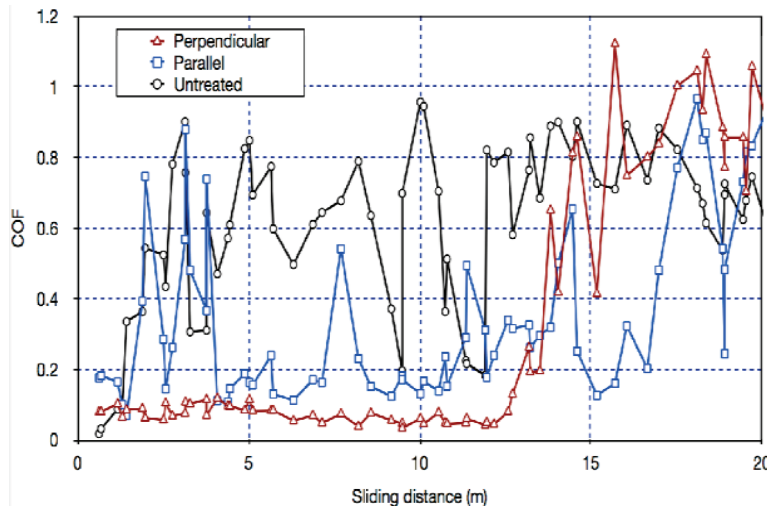

a

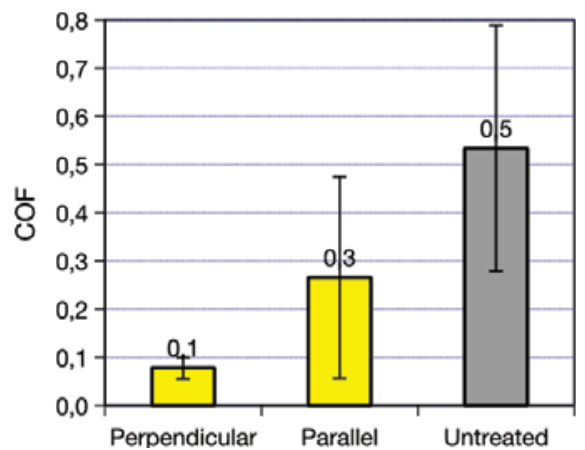

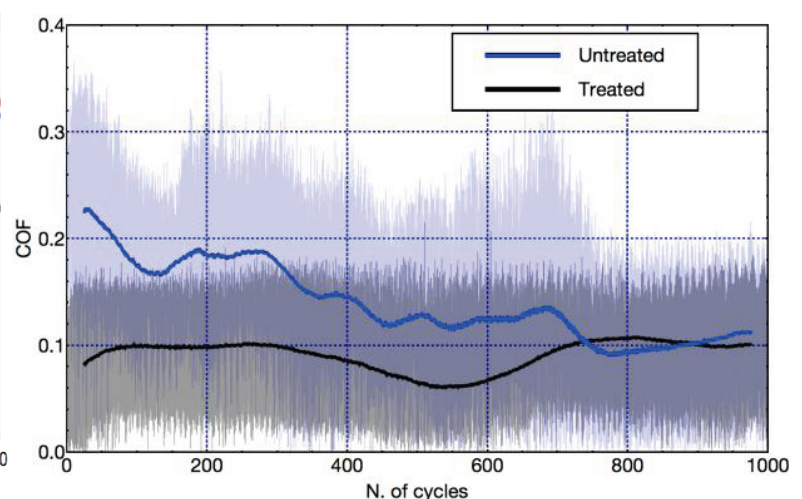

$\mathrm{b}$

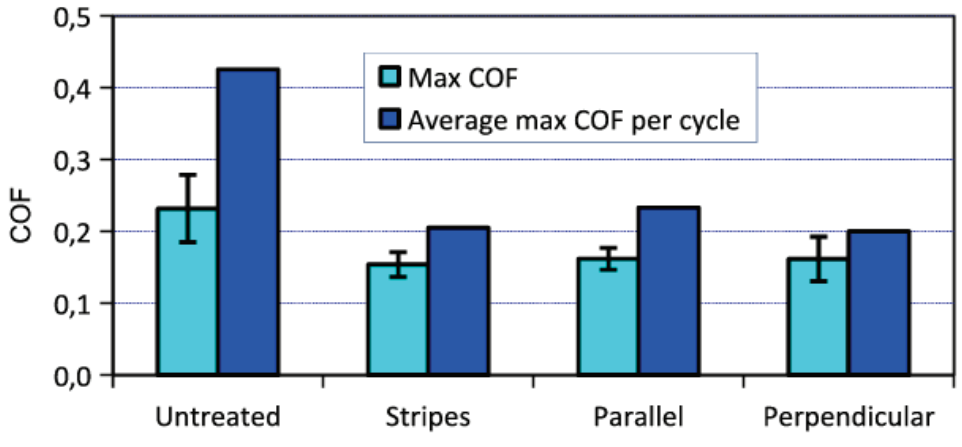

d

Fig. 2. Dry (a) and lubricated (b) sliding tests in real time. Maximum values and average of the maximum coefficient of friction (COF) for dry (c) and lubricated (d) tests, untreated or treated with different orientations of the NLL pattern with respect to the sliding direction

\section{References}

[1] J. Bonse, R. Koter, M. Hartelt, D. Spaltmann, S. Pentzien, Hohm, A. Rosenfeld, J. Kruger, “Femtosecond laser-induced periodic surface structures on steel and titanium alloy for tribological applications," Appl. Phys. A s00339-014-8229-2. (2005).

[2] A.Y. Vorobyev, C. Guo, “Femtosecond laser structuring of titanium implants," j.apsusc. 253 (2007) 7272-7280.

[3] B. Oktem, I. Pavlov, S. Ilday, H. Kalaycıoglu, A. Rybak, S. Yavas, M. Erdogan, \& F. O. Ilday,” Nonlinear laser lithography for indefinitely large-area nanostructuring with femtosecond pulses", Nat. Photonics 7 (2013) 897. 